\title{
Post-thoracotomy pain syndrome and sensory disturbances following thoracotomy at 6- and I2-month follow-ups
}

This article was published in the following Dove Press journal:

Journal of Pain Research

21 March 2017

Number of times this article has been viewed

\author{
Fredrik Hetmann' \\ Ulf E Kongsgaard ${ }^{2,3}$ \\ Leiv Sandvik ${ }^{4}$ \\ Inger Schou-Bredal ${ }^{3,5}$ \\ 'Department of Nursing and \\ Health Promotion, Faculty of \\ Health Sciences, Oslo and Akershus \\ University College of Applied \\ Sciences, ${ }^{2}$ Division of Emergencies \\ and Critical Care, Department of \\ Anaesthesiology, Oslo University \\ Hospital, ${ }^{3}$ Medical Faculty, University \\ of Oslo, ${ }^{4}$ Department of Biostatistics \\ and Epidemiology, Division of Oslo \\ Hospital Services, ${ }^{5}$ Department \\ of Breast and Endocrine Surgery, \\ Division of Cancer and Surgery, Oslo \\ University Hospital, Oslo, Norway
}

Background: Persistent pain affects a large proportion of patients after thoracotomy and is associated with sensory disturbances. The objective of this prospective study was to investigate the time course of pain and sensory disturbances over a 12-month period.

Methods: Patients scheduled for thoracotomy were recruited. Data were collected on the day before surgery, including baseline characteristics and the presence of any preoperative pain. At 6- and 12-month follow-ups, data on pain were collected using the Brief Pain Inventory-Short Form, and perceived sensory disturbances around the thoracotomy scar were recorded from a self-exploration test.

Results: At 12 months after surgery, 97 patients had complete data including baseline and 6and 12-month measurements. Almost half of the patients reported post-thoracotomy pain at the follow-ups. However, $20 \%$ of the patients not reporting post-thoracotomy pain at 6 months did report it at 12 months. Between $40 \%$ and $60 \%$ of patients experienced some kind of sensory disturbance at 6 months. A small decline in some kind of sensory disturbance was reported by $20 \%-50 \%$ of patients at 12 months.

Conclusion: A proportion of patients experienced either resolved or delayed onset of pain. Sensory changes were strongly associated with post-thoracotomy pain syndrome, but were also present in a large proportion of patients without it.

Keywords: pain, post-thoracotomy pain syndrome, chronic pain, thoracic surgery, sensory disturbance, delayed pain

\section{Introduction}

It is estimated that $10 \%-80 \%$ of patients experience persistent pain beyond 3 months after various surgical procedures. ${ }^{1,2}$ Classification of chronic pain is important in conjunction with research to ensure a standardized timing of when to do follow-up measurements. The International Association for the Study of Pain guidelines suggest that the distinction between acute and chronic nonmalignant pain is most conveniently done at 3 months. However, in conjunction with research classifying chronic pain at 6 months is often preferred.

Post-thoracotomy pain syndrome (PTPS) is defined as pain along the thoracotomy scar recurring or persisting more than 2 months after surgery. ${ }^{3}$ It has been estimated that PTPS affects $30 \%-50 \%$ of patients. ${ }^{2,4,5}$ The emphasis in previous research has been on reporting the prevalence of persisting pain and risk factors. Intercostal nerve injury has been proposed as a cause of PTPS, and previous studies have focused on investigating the neuropathic pain component to understand the underlying mechanisms better. ${ }^{6-10}$ Recent studies have used the expressions "sensory changes" or "disturbances" rather
Correspondence: Fredrik Hetmann Department of Nursing and Health Promotion, Faculty of Health Sciences, Oslo and Akershus University College of Applied Sciences, Postboks 4 St. Olavs plass N-0I30, Oslo, Norway Tel +479322 427I

Email Fredrik.Hetmann@hioa.no 
than "neuropathic pain component". ${ }^{11,12}$ The classification of sensory disturbances might be less strict than the classification of neuropathic pain, so it could apply to more patients. Guastella et $\mathrm{al}^{8}$ found that approximately $30 \%$ of patients with PTPS could be classified as having neuropathic pain. However, in the same group of patients, $66 \%$ reported some sort of sensory deficit. In a recent study by Grosen et al, ${ }^{11} 62 \%$ of patients classified with PTPS reported sensory changes in the painful area. As pointed out by Wildgaard et al, ${ }^{13}$ the heterogeneity of data from studies of PTPS makes it difficult to do consistent comparisons among these studies.

Little attention has been paid in previous studies regarding the pattern of persisting pain and sensory changes. Few studies have explored changes in persistent pain and sensory disturbances over time. Mejdahl et $\mathrm{al}^{14}$ did a 6-year followup of persisting pain and sensory disturbances after breast cancer surgery. Out of 1087 patients reporting persisting pain (defined as pain lasting longer than 3 months after surgery) in 2008, pain had resolved in 389 (36\%) of the patients 6 years later. Out of 1271 pain-free patients in 2008, $185(15 \%)$ reported pain at the 6-year follow-up. The pattern was similar for sensory disturbances: of 1343 patients reporting sensory disturbances in 2008, 331 (25\%) patients no longer reported this at the follow-up. From a sample of 955 patients reporting no sensory disturbances in 2008, 164 (17\%) reported them at the 6-year follow-up. The simultaneous occurrence of pain and sensory disturbances caused by nerve injury is one pathogenic mechanism believed to cause PTPS. ${ }^{6}$ However, sensory disturbances are not always associated with pain. ${ }^{15}$ Trajectories of pain and sensory disturbances have yet to be investigated consistently. To our knowledge, no studies to date have reported the time course of persisting pain and sensory disturbances after thoracotomy. Therefore, the aim of this study was to investigate the time course of pain and sensory disturbances over a 12-month period following thoracotomy.

\section{Materials and methods}

From December 2007 to August 2010, 170 patients scheduled for lung surgery by thoracotomy were recruited consecutively for this prospective study. The number of patients to be included was determined by power estimation performed prior to study start. The inclusion criteria were that patients needed to be over 18 years of age, could speak and write Norwegian, and provided written informed consent. The exclusion criteria were the presence of any cognitive impairment or physical inability to fill out a questionnaire. Approval for this study was obtained in September 2007 from the Regional Committee for Medical Research Ethics in Eastern Norway,
Oslo, Norway (REK no. 420-07197a 1.2007.1165) and Social Science Data Services at Oslo University Hospital (no. 849).

\section{Preoperative assessment}

A questionnaire was administered to all patients on the day before surgery, covering baseline characteristics and the presence of any preoperative pain. The questionnaire consisted of the validated Norwegian edition of the Brief Pain InventoryShort Form (BPI-SF). ${ }^{16,17}$ BPI-SF is a general pain measurement tool consisting of 15 questions mapping pain localization, intensity, treatment, and interference with daily activities. The first question asks whether pain other than everyday kinds of pain (eg, headaches, sprains, or toothache) is present. If the answer is "yes" for the first question, the rest of the questionnaire is completed. Patients then mark the location(s) of the pain on a body map, which is followed by four questions regarding pain severity (least, worst, average, and current pain) on an 11-point scale from 0 (no pain) to 10 (pain as bad as can be imagined). Furthermore, two questions address pain treatment and its effect. The last seven questions address pain interference with everyday activities for the previous $24 \mathrm{~h}$ on an 11-point scale from 0 (does not interfere) to 10 (completely interferes). Results regarding pain intensity, treatment, and interference with daily activities have been reported elsewhere. ${ }^{18}$

\section{Treatment}

Thoracotomies were performed using an open posterolateral technique by a small group of experienced surgeons. Postoperative analgesia was ensured by thoracic epidural analgesia with continuous infusion of a commercially prepared triple drug mixture (Standard EDA blend; Fresenius Kabi, Halden, Norway: bupivacaine, $1 \mathrm{mg} / \mathrm{mL}$; fentanyl, $2 \mu \mathrm{g} / \mathrm{mL}$; and adrenaline, $2 \mu \mathrm{g} / \mathrm{mL}$ ). Patients were weaned off thoracic epidural analgesia on the third or fourth postoperative day. Paracetamol (1 g) was given routinely every $6 \mathrm{~h}$. Breakthrough pain was treated with intravenous ketobemidone (Ketorax; Pfizer, Lysaker, Norway). Postoperative pain was assessed for six consecutive days after the surgery, or as long as the patients were hospitalized, as reported elsewhere. ${ }^{19}$

\section{Assessment at 6- and 12-month follow-ups}

At 6- and 12-month follow-ups, questionnaires were sent by mail to all enrolled patients, after consulting the Norwegian National Registry for patients who might have died in the period. In addition to the BPI-SF, the participants were also asked to do a self-exploration sensory test described by Romundstad et $\mathrm{al}^{20}$ of the area around the thoracotomy scar. The purpose was to determine any subjective increased or 
decreased sensitivity around the scar. The participants were asked to stroke a finger over an area from below the scar and upward, comparing the sensation with the skin on the abdomen. Increased or decreased sensitivity was indicated with either "yes" or "no". Furthermore, the participants were asked to rate any pain with normal touching, pressure, cold, warmth, or from clothes/showering on a 4-point numeric rating scale $(0=$ no, $1=$ mild, $2=$ moderate, and $3=$ strong $)$. The self-exploration sensory test was modified to suit the surgical technique for this study, after permission had been obtained from Romundstad et al.

Patients reporting pain - other than an everyday kind associated with the surgical area as indicated on the body map were categorized as having PTPS at 6 and/or 12 months. Medical records for all patients reporting persistent pain at 12 months were examined to exclude other causes that could explain persistent pain. None was found.

\section{Statistical analysis}

Statistical analyses were performed using IBM SPSS statistics version 23.0 for Windows (IBM Corp., Armonk, NY, USA). Descriptive statistics, frequencies and (percentages), and mean and ( \pm standard deviations) were utilized. We compared reported sensory changes in the non-PTPS and PTPS groups using Fisher's exact test (for $2 \times 2$ contingency tables). The level of significance was set at $p<0.05$.

\section{Results}

One-hundred and seventy patients were included at baseline. At 6 months, 118 had returned the questionnaire, with 19 dead and 33 not responding. One year after surgery, 97 of the questionnaires (57\%) had complete data including baseline and 6- and 12-month follow-up information. In total, 73 patients $(43 \%)$ were lost to follow-up because of no reply or death (Figure 1). The mean age of the 97 participants was 64.2 years $( \pm 10.6)$, and $42(43 \%)$ were female.

\section{Pain at baseline, 6, and 12 months}

Thirty-two patients $(33 \%)$ reported preoperative pain at baseline. In total, 48 (49\%) patients reported persistent pain at 6 months. Among these, $24(50 \%)$ had also reported preoperative pain. At 12 months, persistent pain was reported by 47 (48\%) patients. Twenty-three (48\%) of these patients had also reported preoperative pain. Furthermore, of the $49(50 \%)$ patients not reporting persistent pain at 6 months, $10(20 \%)$ reported persistent pain at 12 months. Among the 48 patients (49\%) reporting persistent pain at 6 months, 11 (11\%) no longer reported it at 12 months (Figure 2).

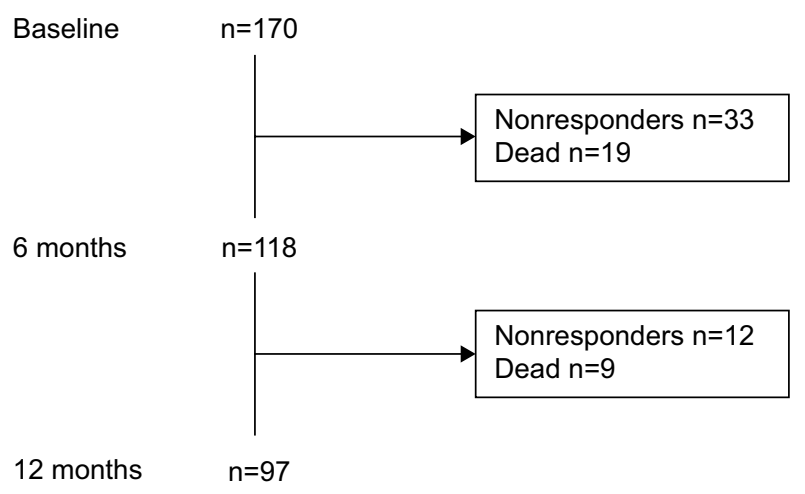

Figure I Flowchart of patient inclusion and losses.

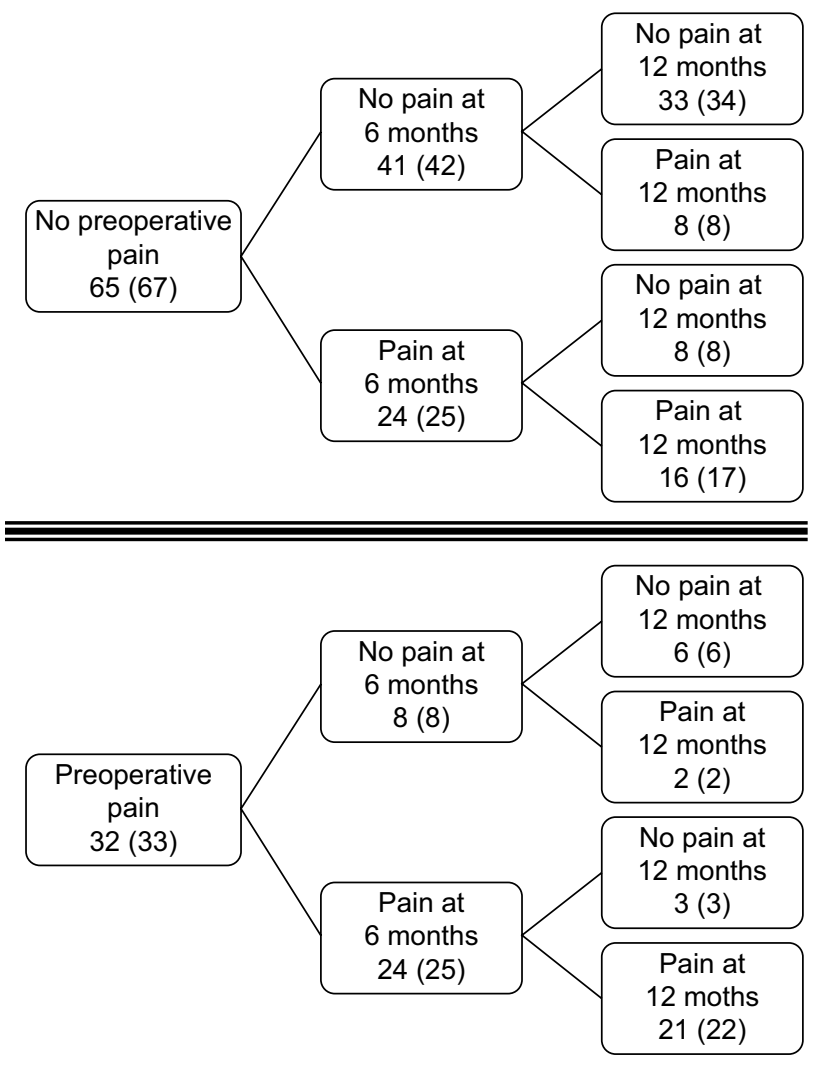

Figure 2 Changes in persistent pain from 6 to 12 months in patients with or without preoperative pain. Numbers are presented as $\mathrm{n}$ and (\%).

\section{Self-reported sensory disturbances}

At 6 months, self-reported sensory disturbances were distributed as follows: 40 patients $(51 \%)$ reported increased sensitivity, $60(62 \%)$ had pain with normal touching, 56 (58\%) pain with pressure, $39(40 \%)$ pain with cold, $38(39 \%)$ pain with warmth, 48 (49\%) pain with clothes/showering, and $44(45 \%)$ reported decreased sensitivity. Among those reporting painful sensory disturbances, 15 (16\%) reported strong pain with normal touching, $21(22 \%)$ strong pain with pressure, 4 (4\%) strong pain with cold, 1 (1\%) strong pain with warmth, and $10(10 \%)$ strong pain with clothes/ 
showering. At 12 months, 47 (49\%) reported increased sensitivity, 48 (50\%) pain with normal touching, 39 (40\%) pain with pressure, $24(25 \%)$ pain with cold, $20(21 \%)$ pain with warmth, 34 (35\%) pain with clothes/showering, and 44 (45\%) reported decreased sensitivity. Among those reporting painful sensory disturbances, $9(9 \%)$ reported strong pain with normal touching, $10(10 \%)$ strong pain with pressure, $8(8 \%)$ strong pain with cold, 3 (3\%) strong pain with warmth, and 6 (6\%) strong pain with clothes/showering. Increased sensitivity, pain with normal touching, and decreased sensitivity were significantly more predominant in patients reporting PTPS at 6 months (Table 1). At 12 months, those patients reporting PTPS also reported significantly more increased sensitivity, pain with normal touching, pain with pressure, pain with clothes/showering, and decreased sensitivity, compared with patients not reporting PTPS.

\section{Discussion}

A third of the patients in this study reported preoperative pain. Almost half of them reported persistent pain at 6- and 12-month follow-ups after thoracotomy. Among those patients reporting PTPS at 6 months, 11\% no longer reported it at 12 months. Somewhat surprisingly, $20 \%$ of patients who did not report PTPS at 6 months did report it at 12 months. However, we did not observe any statistically significant change in the

Table I Cross tabulation of self-reported sensory disturbances in patients without PTPS or with PTPS 6- and 12-month followups after thoracotomy

\begin{tabular}{|c|c|c|c|}
\hline $\begin{array}{l}\text { Sensory } \\
\text { disturbances }\end{array}$ & $\begin{array}{l}\text { Patients } \\
\text { without } \\
\text { PTPS, n (\%) }\end{array}$ & $\begin{array}{l}\text { Patients with } \\
\text { PTPS, n (\%) }\end{array}$ & $p$-value* \\
\hline \multicolumn{4}{|l|}{6 months } \\
\hline Increased sensitivity & $13(35)$ & $27(66)$ & 0.01 \\
\hline \multicolumn{4}{|c|}{ Increased sensitivity or pain with: } \\
\hline Touch & $23(47)$ & 37 (79) & 0.002 \\
\hline Pressure & $24(49)$ & $32(66)$ & ns \\
\hline Cold & $19(39)$ & $20(42)$ & ns \\
\hline Warmth & $19(39)$ & $19(40)$ & ns \\
\hline Clothes/shower & $20(4 I)$ & $28(58)$ & ns \\
\hline Decreased sensitivity & $15(34)$ & $29(63)$ & 0.007 \\
\hline \multicolumn{4}{|l|}{12 months } \\
\hline Increased sensitivity & $19(4 I)$ & $28(64)$ & 0.04 \\
\hline \multicolumn{4}{|c|}{ Increased sensitivity or pain with: } \\
\hline Touch & $19(38)$ & $29(62)$ & 0.03 \\
\hline Pressure & I4 (28) & $25(53)$ & 0.01 \\
\hline Cold & $10(20)$ & I $4(30)$ & ns \\
\hline Warmth & $8(16)$ & $12(26)$ & ns \\
\hline Clothes/shower & $12(24)$ & $22(47)$ & 0.02 \\
\hline Decreased sensitivity & $17(35)$ & $27(60)$ & 0.02 \\
\hline
\end{tabular}

Note: *Fisher's exact test for $2 \times 2$ contingency table.

Abbreviations: ns, not significant; PTPS, post-thoracotomy pain syndrome. prevalence of PTPS between 6- and 12-month follow-ups. Between $40 \%$ and $60 \%$ of patients experienced some kind of sensory disturbance at 6 months. A small decline in the prevalence of sensory disturbance was observed at 12 months.

When comparing different studies on chronic postsurgical pain, the timing of follow-ups is critical. This has been emphasized by VanDenKerkhof et al, who advocated standardization for epidemiological studies on pain. ${ }^{21}$ Their review identified inequalities in the timing and definition of chronic postsurgical pain, with follow-ups of 2-6 months. Prospective longitudinal studies are often time-consuming, if the follow-up exceeds 6 months. Defining chronic postsurgical pain at 3 or 6 months is a pragmatic approach based more on practical than on scientific considerations. The literature on pain changes beyond 12 months is inadequate, because most studies do not extend past this time. One important factor in the present study was the high mortality for patients with lung cancers. Estimates from the Norwegian Cancer Registry state that the 5-year relative survival rate is $13.2 \%$ for men and $19.2 \%$ for women. ${ }^{22}$ Some studies have reported chronic postsurgical pain to be prevalent in $13 \%-35 \%$ of patients between 2 and 10 years after thoracotomy. ${ }^{11,15,23}$ However, pain trajectories for these patients have not been reported.

In this study, approximately half of the patients reported PTPS at 6- and 12-month follow-ups. This is in contrast to a study by Grosen et al, ${ }^{11}$ which found pain prevalence to be only around $20 \%$. By reporting only prevalence, it might seem that only one patient experienced improvement in the present study. One could argue that 6 months is too short to see real improvements in more patients. Reinpold et $\mathrm{al}^{24}$ reported a small decline in chronic pain rate between 6 months and 5 years in a 5-year follow-up of patients after groin hernia repair. At 6 months, the reported chronic pain rate was $16.5 \%$, with little change to $16.1 \%$ at 5 years. Based on these numbers, it seems that chronic pain had resolved in a small number of patients. When looking closer at changes in pain between 6- and 12-month follow-ups, half of the patients in our study reported PTPS at 6 months. At 12 months, PTPS had resolved in eleven of the patients, whereas ten patients reported a delayed onset of PTPS at 12 months. Our findings are in accordance with the results from other studies. ${ }^{11,24} \mathrm{By}$ reporting only the prevalence of PTPS, important information about pain changes might not be elucidated properly. To our knowledge, late-onset PTPS has not been reported in any prospective study. Delayed onset of pain has been described after strokes, spinal cord lesions, limb amputations, hernia repair surgery, and breast cancer surgery. ${ }^{1,14,24}$ Knowledge regarding 
the mechanisms behind these phenomena is scarce. Some reports have suggested that nervous pain memory, threshold triggering factors, slow anatomic changes, physiologic changes, biochemical changes, and axonal nerve damage might be implicated in the delayed onset of pain. ${ }^{1,25}$

Preoperative pain has been found to be a predictor for PTPS. ${ }^{2,4}$ This was further highlighted by following changes in pain for our patients. There seem to be different types of pain changes depending on whether preoperative pain was present. Most of the patients without preoperative pain were also pain free at 12 months. In contrast, most of the patients with preoperative pain also reported pain at 12 months. Over the last decade, evidence from functional imaging studies has suggested that both functional and structural changes in the brain can be found in patients with chronic pain. ${ }^{26,27}$ This might be one plausible explanation of why most of our patients with existing preoperative pain also reported PTPS at 12 months.

The correlation between persistent postsurgical pain and neuropathic pain has been investigated in a growing body of literature over the last decade. Studies have identified neuropathic pain in a large number of patients with PTPS. In a systematic review, Haroutiunian et al found the prevalence of neuropathic pain to be as high as $66 \%$ among patients reporting PTPS. ${ }^{9}$ Other studies have reported prevalences ranging from $12 \%$ to $32 \% .^{5,8,28-30} \mathrm{~A}$ few studies have indicated that the use of a rib retractor might be responsible for intercostal nerve injury during thoracotomy. ${ }^{10,31,32}$ In a small-sample study, Rogers et al identified mechanically induced nerve injury in all patients as a direct consequence of this. ${ }^{32}$ However, not all patients experience persisting pain. Equally common are sensory changes without persisting pain. ${ }^{1,10,31,32}$ Here, we found that patients with PTPS reported sensory changes more frequently. Increased sensitivities to touch and pressure were most commonly reported at both follow-up time points. Interestingly, almost half of the patients without PTPS reported increased sensitivity to touch and pressure at 6 months, but this declined at 12 months. Our results are consistent with previous studies on thoracotomy. Maguire et $\mathrm{al}^{33}$ performed a retrospective survey to assess the neuropathic component of chronic pain, among other factors. Their questionnaire was specially designed, with questions related to modalities associated with neuropathy. They found that "neuropathic symptoms" were reported by $35 \%-82 \%$ of patients and sensory changes were more prevalent in patients with chronic pain. In a more recent study, Wildgaard et $\mathrm{l}^{15}$ found that $63 \%$ of patients with PTPS reported sensory changes. Sensory changes were reported by $25 \%$ of patients without PTPS.

\section{Strengths and limitations}

The strength of this study was its prospective design, with follow-ups at 6 and 12 months. Furthermore, to our knowledge, this is the first prospective study to report changes in pain and sensory disturbances - not merely prevalence rates - over a defined time period after thoracotomy. However, our study had some limitations. First, the rate of patient loss to follow-up was higher than anticipated, resulting in low numbers in several of the categories presented above. Second, even though detailed instructions on how to selfassess were presented in the posted questionnaire, we cannot be certain that this was done according to the instructions. Furthermore, no detailed instructions on how to assess pain with cold or warmth were provided. Thus, the results regarding pain provoked by cold and warmth should be interpreted with caution. Compared to a clinical Quantitative Sensory Test, the self-exploration test in this current study is less sophisticated. However, the self-exploration test is in large part based on properties from the Quantitative Sensory Test.

\section{Conclusion}

Our results suggest that pain changes cannot be treated as linear states, but are dynamic in nature. A proportion of the patients experienced either resolved or delayed onset of pain. Important information regarding pain changes, which may play an important part in further understanding persistent pain, might not become apparent if only the prevalence of PTPS is reported. Sensory changes were strongly associated with PTPS but were also present in a large proportion of patients without it.

\section{Acknowledgment s}

This work was supported by the Department of Research and Development, Division of Emergencies and Critical Care, Oslo University Hospital, Norway and Oslo and Akershus University College of Applied Sciences, Faculty of Health Sciences, Department of Nursing and Health Promotion, Oslo, Norway.

This study was carried out at Oslo University HospitalUllevål, Oslo, Norway.

\section{Disclosure}

The authors report no conflicts of interest in this work.

\section{References}

1. Borsook D, Kussman BD, George E, Becerra LR, Burke DW. Surgically induced neuropathic pain: understanding the perioperative process. Ann Surg. 2013;257(3):403-412. 
2. Kehlet $\mathrm{H}$, Jensen TS, Woolf CJ. Persistent postsurgical pain: risk factors and prevention. Lancet. 2006;367(9522):1618-1625.

3. Merskey H, Bogduk H, editors. Classification of Chronic Pain. 2nd ed. Seattle, WA: IASP Press; 1994.

4. Hetmann F, Kongsgaard UE, Sandvik L, Schou-Bredal I. Prevalence and predictors of persistent post-surgical pain 12 months after thoracotomy. Acta Anaesthesiol Scand. 2015;59(6):740-748.

5. Mongardon N, Pinton-Gonnet C, Szekely B, Michel-Cherqui M, Dreyfus JF, Fischler M. Assessment of chronic pain after thoracotomy: a 1-year prevalence study. Clin J Pain. 2011;27(8):677-681.

6. Doan LV, Augustus J, Androphy R, Schechter D, Gharibo C. Mitigating the impact of acute and chronic post-thoracotomy pain. J Cardiothorac Vasc Anesth. 2014;28(4):1048-1056.

7. Duale C, Guastella V, Morand D, et al. Characteristics of the neuropathy induced by thoracotomy: a 4-month follow-up study with psychophysical examination. Clin J Pain. 2011;27(6):471-480.

8. Guastella V, Mick G, Soriano C, et al. A prospective study of neuropathic pain induced by thoracotomy: incidence, clinical description, and diagnosis. Pain. 2011;152(1):74-81.

9. Haroutiunian S, Nikolajsen L, Finnerup NB, Jensen TS. The neuropathic component in persistent postsurgical pain: a systematic literature review. Pain. 2013;154(1):95-102.

10. Maguire MF, Latter JA, Mahajan R, Beggs FD, Duffy JP. A study exploring the role of intercostal nerve damage in chronic pain after thoracic surgery. Eur J Cardiothorac Surg. 2006;29(6):873-879.

11. Grosen K, Laue PG, Pfeiffer-Jensen M, Hoejsgaard A, Pilegaard HK. Persistent post-surgical pain following anterior thoracotomy for lung cancer: a cross-sectional study of prevalence, characteristics and interference with functioning. Eur J Cardiothorac Surg. 2013;43(1):95-103.

12. Johansen A, Schirmer H, Nielsen CS, Stubhaug A. Persistent postsurgical pain and signs of nerve injury: the Tromsø Study. Acta Anaesthesiol Scand. 2016;60(3):380-392.

13. Wildgaard K, Ravn J, Kehlet H. Chronic post-thoracotomy pain: a critical review of pathogenic mechanisms and strategies for prevention. Eur J Cardiothorac Surg. 2009;36(1):170-180.

14. Mejdahl MK, Andersen KG, Gartner R, Kroman N, Kehlet H. Persistent pain and sensory disturbances after treatment for breast cancer: six year nationwide follow-up study. BMJ. 2013;346:f1865.

15. Wildgaard K, Ravn J, Nikolajsen L, Jakobsen E, Jensen TS, Kehlet H. Consequences of persistent pain after lung cancer surgery: a nationwide questionnaire study. Acta Anaesthesiol Scand. 2011;55(1):60-68.

16. Cleeland CS, Ryan KM. Pain assessment: global use of the Brief Pain Inventory. Ann Acad Med Singapore. 1994;23(2):129-138.

17. Klepstad P, Loge JH, Borchgrevink PC, Mendoza TR, Cleeland CS, Kaasa $S$. The Norwegian brief pain inventory questionnaire: translation and validation in cancer pain patients. J Pain Symptom Manage. 2002;24(5):517-525.

18. Hetmann F, Kongsgaard UE, Sandvik L, Schou-Bredal I. Prevalence and predictors of persistent post-surgical pain 12 months after thoracotomy. Acta Anaesthesiol Scand. 2015;59(6):740-748.
19. Hetmann F, Schou-Bredal I, Sandvik L, Kongsgaard UE. Does chronic pre-operative pain predict severe post-operative pain after thoracotomy? A prospective longitudinal study. Acta Anaesthesiol Scand. 2013;57(8): 1065-1072.

20. Romundstad L, Breivik H, Roald H, Skolleborg K, Romundstad PR, Stubhaug A. Chronic pain and sensory changes after augmentation mammoplasty: long term effects of preincisional administration of methylprednisolone. Pain. 2006;124(1-2):92-99.

21. VanDenKerkhof EG, Peters ML, Bruce J. Chronic pain after surgery: time for standardization? A framework to establish core risk factor and outcome domains for epidemiological studies. Clin J Pain. 2013;29(1): 2-8.

22. Norwegian National Cancer Registry; 2016. Available from: https:// www.kreftregisteret.no/. Accessed May 20, 2016.

23. Montes A, Roca G, Sabate S, et al. Genetic and clinical factors associated with chronic postsurgical pain after hernia repair, hysterectomy, and thoracotomy: a two-year multicenter cohort study. Anesthesiology. 2015;122(5):1123-1141.

24. Reinpold WM, Nehls J, Eggert A. Nerve management and chronic pain after open inguinal hernia repair: a prospective two phase study. Ann Surg. 2011;254(1):163-168.

25. Jaaskelainen SK, Teerijoki-Oksa T, Virtanen A, Tenovuo O, Forssell $\mathrm{H}$. Sensory regeneration following intraoperatively verified trigeminal nerve injury. Neurology. 2004;62(11):1951-1957.

26. Borsook D, Erpelding N, Becerra L. Losses and gains: chronic pain and altered brain morphology. Expert Rev Neurother. 2013;13(11): $1221-1234$

27. May A. Chronic pain may change the structure of the brain. Pain. 2008; 137(1):7-15.

28. Duale C, Ouchchane L, Schoeffler P, Dubray C; EDONIS Investigating Group. Neuropathic aspects of persistent postsurgical pain: a French multicenter survey with a 6-month prospective follow-up. J Pain. 2014; 15(1):24.e1-24.e20.

29. Johansen A, Romundstad L, Nielsen CS, Schirmer H, Stubhaug A. Persistent postsurgical pain in a general population: prevalence and predictors in the Tromsø Study. Pain. 2012;153(7):1390-1396.

30. Peng Z, Li H, Zhang C, Qian X, Feng Z, Zhu S. A retrospective study of chronic post-surgical pain following thoracic surgery: prevalence, risk factors, incidence of neuropathic component, and impact on quality of life. PLoS One. 2014;9(2):e90014.

31. Benedetti F, Vighetti S, Ricco C, et al. Neurophysiologic assessment of nerve impairment in posterolateral and muscle-sparing thoracotomy. J Thorac Cardiovasc Surg. 1998;115(4):841-847.

32. Rogers ML, Henderson L, Mahajan RP, Duffy JP. Preliminary findings in the neurophysiological assessment of intercostal nerve injury during thoracotomy. Eur J Cardiothorac Surg. 2002;21(2):298-301.

33. Maguire MF, Ravenscroft A, Beggs D, Duffy JP. A questionnaire study investigating the prevalence of the neuropathic component of chronic pain after thoracic surgery. Eur J Cardiothorac Surg. 2006;29(5): 800-805.
Journal of Pain Research

\section{Publish your work in this journal}

The Journal of Pain Research is an international, peer reviewed, open access, online journal that welcomes laboratory and clinical findings in the fields of pain research and the prevention and management of pain. Original research, reviews, symposium reports, hypothesis formation and commentaries are all considered for publication.

\section{Dovepress}

The manuscript management system is completely online and includes a very quick and fair peer-review system, which is all easy to use. Visit http://www.dovepress.com/testimonials.php to read real quotes from published authors. 